\title{
An automatic method for identifying surface proteins in bacteria: SLEP
}

Emanuela Giombini', Massimiliano Orsini ${ }^{2}$, Danilo Carrabino ${ }^{3}$, Anna Tramontano ${ }^{1,4^{*}}$

\begin{abstract}
Background: Bacterial infections represent a global health challenge. The identification of novel antibacterial targets for both therapy and vaccination is needed on a constant basis because resistance continues to spread worldwide at an alarming rate. Even infections that were once easy to treat are becoming difficult or, in some cases, impossible to cure. Ideal targets for both therapy and vaccination are bacterial proteins exposed on the surface of the organism, which are often involved in host-pathogen interaction. Their identification can greatly benefit from technologies such as bioinformatics, proteomics and DNA microarrays.

Results: Here we describe a pipeline named SLEP (Surface Localization Extracellular Proteins), based on an automated optimal combination and sequence of usage of reliable available tools for the computational identification of the surfome, i.e. of the subset of proteins exposed on the surface of a bacterial cell.

Conclusions: The tool not only simplifies the usage of these methods, but it also improves the results by selecting the specifying order and combination of the instruments. The tool is freely available at http://www.caspur.it/slep.
\end{abstract}

\section{Background}

Bacterial diseases are among the major causes of mortality and morbidity in humans. Antibiotics are the first line of defence against bacteria, however more and more bacteria are antibiotic resistant and the phenomenon is spreading at an alarming rate [1,2]. Many diseases are becoming increasingly difficult to fight. There are several examples of microbial infections that are becoming resistant to all existing therapies and for which a vaccination strategy is deemed to be appropriate, such as gonorrhea, tuberculosis, pneumonia, septicaemia and childhood ear infections [3-7].

Among the proteins encoded by bacteria, secreted and surface proteins are particularly important in bacterial pathogenesis. The former can be involved in host cell toxicity and lead to more or less subtle alterations of the host cell for the benefit of the pathogen. Bacterial surface proteins play a fundamental role in the interaction with the cell environment [8-12]. They can be involved in adhesion and invasion of the host cells as well as in defending against host responses. Because of this, surface proteins are potential drug targets [13].

\footnotetext{
* Correspondence: anna.tramontano@uniroma1.it
'Department of Biochemical Sciences "A. Rossi Fanelli", Sapienza University.

* Correspondence: anna.tramontano@uniroma1.it
${ }^{1}$ Department of Biochemical Sciences "A. Rossi Fanelli", Sapienza University. 00185 Rome, Italy
} (185 Rome, laly

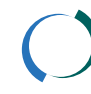

Moreover, surface proteins are likely to interact with the host immune system and are ideal candidates for vaccine development [14-16].

Surface proteins include integral or transmembrane proteins that span the membrane and have a hydrophilic cytosolic domain, which interacts with internal molecules, a hydrophobic membrane-spanning domain that anchors it within the cell membrane, and a hydrophilic extracellular domain that interacts with external molecules. Lipid anchored proteins are instead covalentlybound to one or more lipid molecules. Other membrane proteins are peripheral, i.e. they are attached to integral membrane proteins, or associated with regions of the lipid bilayer.

Gram-positive bacteria possess a thick cell wall containing many layers of peptidoglycan and teichoic acids. In contrast, Gram-negative bacteria have a relatively thin cell wall consisting of a few layers of peptidoglycan surrounded by a second lipid membrane containing lipopolysaccharides and lipoproteins. This is reflected in their membrane protein composition. Cell wall proteins are found in Gram+ bacteria while $\beta$-barrel membrane proteins are only found in the outer membranes of Gram- organisms, in mitochondria and chloroplast [17]. 
Despite the biological relevance of bacterial surface proteins, their characterization is still incomplete. There are two main routes to identify surface proteins. In one approach, membrane and cell wall fractions are separated from the cytoplasmic fraction and then proteins are identified by two-dimensional (2D)-electrophoresis or 2D-chromatography coupled to mass spectrometry [see for example [18-23]]. The other possibility is to take advantage of bioinformatics and attempt their prediction on the basis of one of the many specifically developed algorithms.

There is a plethora of available tools for predicting the membrane localization and topology of a protein and the presence of specific localization signals in its sequence, but not every method is equally accurate and, especially, an end user is not always well informed about novel developments in the field. The order in which these tools are used might also make a difference, as we will show here. Furthermore, each of them tends to use different input formats and not always self explanatory output formats.

The aim of the work described here is to bring these tools in a coordinated and easy-to use form to the bench scientists who, on one side, should not need to be familiar with the ins and outs of each and every tool, but, on the other, should be given sufficient information to assess the reliability of the methods.

\section{Implementation}

SLEP and all the related tools have been implemented locally on a linux SLES 10 server.

The programs included in the SLEP automatic procedure are Glimmer [24-26], TMHMM [27,28], prodivHMM [29,30], pSORTb [31,32] and LipoP [30] all ran with default parameters.

If the user inputs a genome, putative genes need to be identified and translated into their amino acid sequence. This is achieved using Glimmer, a gene finding program based on Interpolated Markov Models (IMMs) [24-26]. The accuracy of gene identification by Glimmer depends upon the length and the GC-content the genome and is reported at http://www.cbcb.umd.edu/software/glimmer/.

The translated gene products, or the input proteins (if the user selected to start with a known proteome) are analysed for the presence of transmembrane regions using TMHMM [27,28] and prodiv-HMM [29], two independent Hidden Markov Model-based prediction methods. It is a known problem in the field that signal peptides might often be mispredicted as transmembrane helices and vice versa. To alleviate this pro- blem, we only assign the "membrane protein" tag to proteins for which more than three transmembrane helix are predicted by at least one method. As described later, proteins for which no signal peptide is identified are re-submitted to the transmembrane prediction tools.

Proteins not assigned to the "membrane" bin are analysed using LipoP [30], a tool for identifying signal peptides of both type I and II in a protein sequence. Because all clearly detectable membrane proteins have been already filtered out in the previous step, the number of false positives, i.e. the number of times LipoP predicts as a signal peptide what is in reality a transmembrane helix, is reduced. Table 1 shows the comparison between the accuracy obtained using LipoP on the complete dataset and that achieved by running it only on the filtered set of proteins, i.e. on proteins not including predicted transmembrane proteins with three or more helices, according to the SLEP protocol. The decrease in the number of false positives, although rather small, justifies our choice in using the tool only after filtering out the predicted multiple membrane spanning proteins.

The next step consists in running pSORTb [31,32] on the remaining set of proteins. pSORTb is used for recognizing cell wall proteins (in Gram + bacteria) and outer membrane proteins (in Gram-bacteria) as well as exported proteins. The remaining proteins are reanalysed by TMHMM and prodiv-HMM in order to identify proteins with a single membrane spanning helix. As mentioned before, we remove clearly detectable membrane proteins before attempting the prediction of the presence of signal peptides. Only if no signal peptide has been identified in the sequence, we look for single membrane spanning helix.

The statistical parameters used for evaluating the accuracy of the predictions are:

Table 1 Comparison between SLEP and LipoP

\begin{tabular}{|c|c|c|c|}
\hline & TP & FP & $\mathrm{FN}$ \\
\hline \multicolumn{4}{|l|}{ Gram+ } \\
\hline LipoP & 227 & 47 & 5 \\
\hline LipoP/SLEP & 227 & 19 & 5 \\
\hline \multicolumn{4}{|l|}{ Gram- } \\
\hline LipoP & 266 & 45 & 5 \\
\hline LipoP/SLEP & 266 & 43 & 5 \\
\hline
\end{tabular}

Comparison of the accuracy of LipoP [30] ran on the whole set of proteins and on a reduced set after removing predicted transmembrane proteins with three or more helices according to the SLEP protocol. 


$$
\begin{aligned}
& \text { Accuracy }=\frac{T P+T N}{T P+F P+F N+T N} \times 100 \\
& \text { Specficity }(S P)=\frac{T N}{T N+F P} \times 100 \\
& \text { Sensitivity }(S E)=\frac{T P}{T P+F N} \times 100 \\
& \text { Positive predictive value }(P P V)=\frac{T P}{T P+F P} \times 100 \\
& \text { Negative predictive value }(N P V)=\frac{T N}{T N+F N} \times 100 \\
& \text { Matthews Correlation Coefficient }(M C C)= \\
& =\frac{T P \times T N-F P \times F N}{(T P+F P) \times(T P+F N) \times(T N+F P) \times(T N+F N)} \times 1 C
\end{aligned}
$$

Where TP, TN, FP and FN are the number of True Positive, True Negative, False Positive and False Negative results, respectively.

\section{Results and discussion}

SLEP is based on an automated optimal combination and succession of usage of some of the most reliable available tools. The user needs to input either the genomic sequence of the bacterial organisms under study or its proteome together with the information of whether the bacterium is Gram+ or Gram-. The main purpose of SLEP is to provide users with an easy-to-use tool for the prediction of protein localization with the highest possible accuracy achievable today. The user interface of the tool is illustrated in Figure 1.
The output of the system is an organized list of proteins (or putative proteins if the input is a genome) classified according to their predicted localization. In particular it will separately list lipoproteins, membrane, exported and secreted proteins, cell wall proteins or outer membrane proteins in Gram+ or Gram- bacteria, respectively (Figure 2).

We tested the accuracy of the procedure using the manually curated database Swiss Prot. This dataset, named SP, contained 18,510 protein sequences of known localization (as reported in the SUBCELLULAR LOCATION field), roughly equally populated by protein 100from Gram- and Gram+ bacteria (9,946 and 9,564, respectively). For Gram + bacteria we used Enterococcus (EN, 228 proteins), Listeria (LI, 749 proteins), Staphylococcus (SP, 3981 proteins), Streptococcus (ST, 179 proteins) and a pool of Bacilli Gram+ organisms (B+, 4427 proteins). For Gram-, the datasets contained data from E. coli (EC, 3891 proteins), Legionella (LE, 421 proteins), Pseudomonas (PS, 3369 proteins), Salmonella (SA, 2019 proteins) and a pool of Bacilli Gram- organisms (B-, 246 proteins).

The overall accuracy of the predictions that can be achieved in a single click using SLEP is illustrated in Table 2.

In Table 3 we compare our results with the use of PSORTb alone. For completeness, we report in the same Table the accuracy of other available methods for the relevant datasets [33-38]. Notice that the tools included in SLEP have been selected for their accuracy, but also

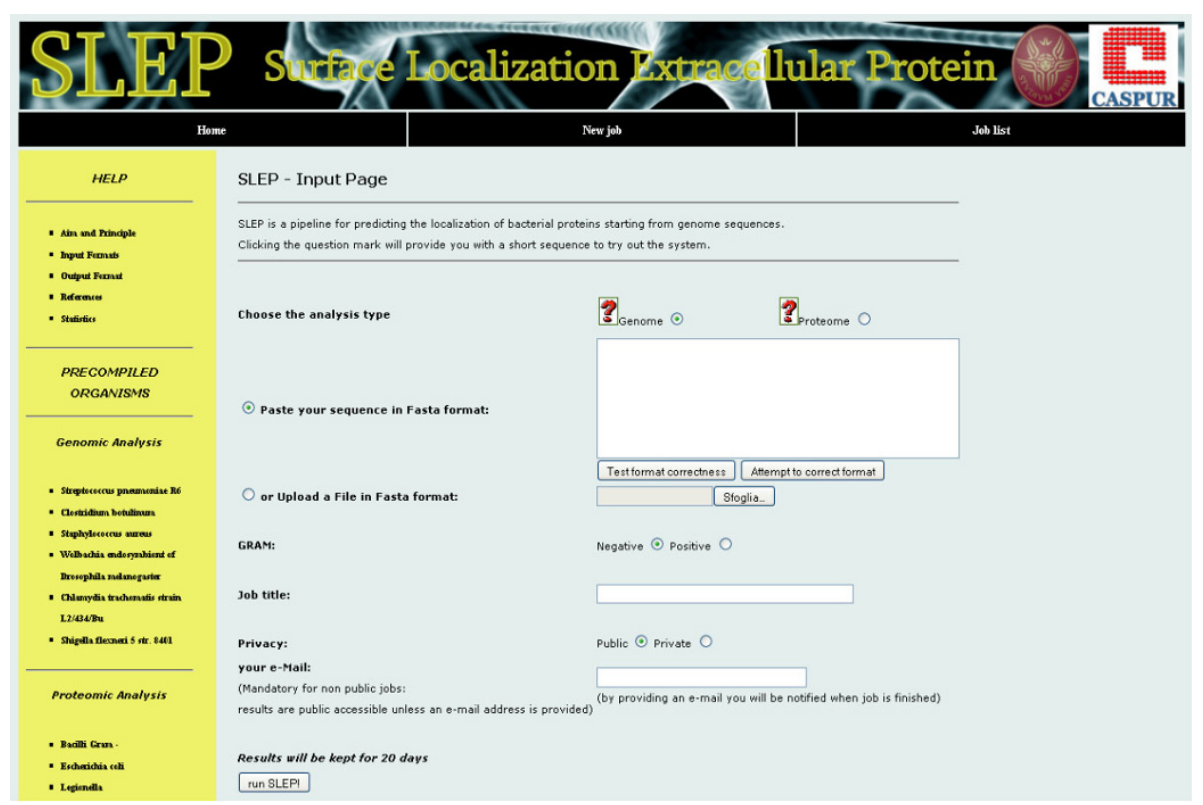

Figure 1 SLEP home page. Graphics interface of the initial submission page of SLEP. 


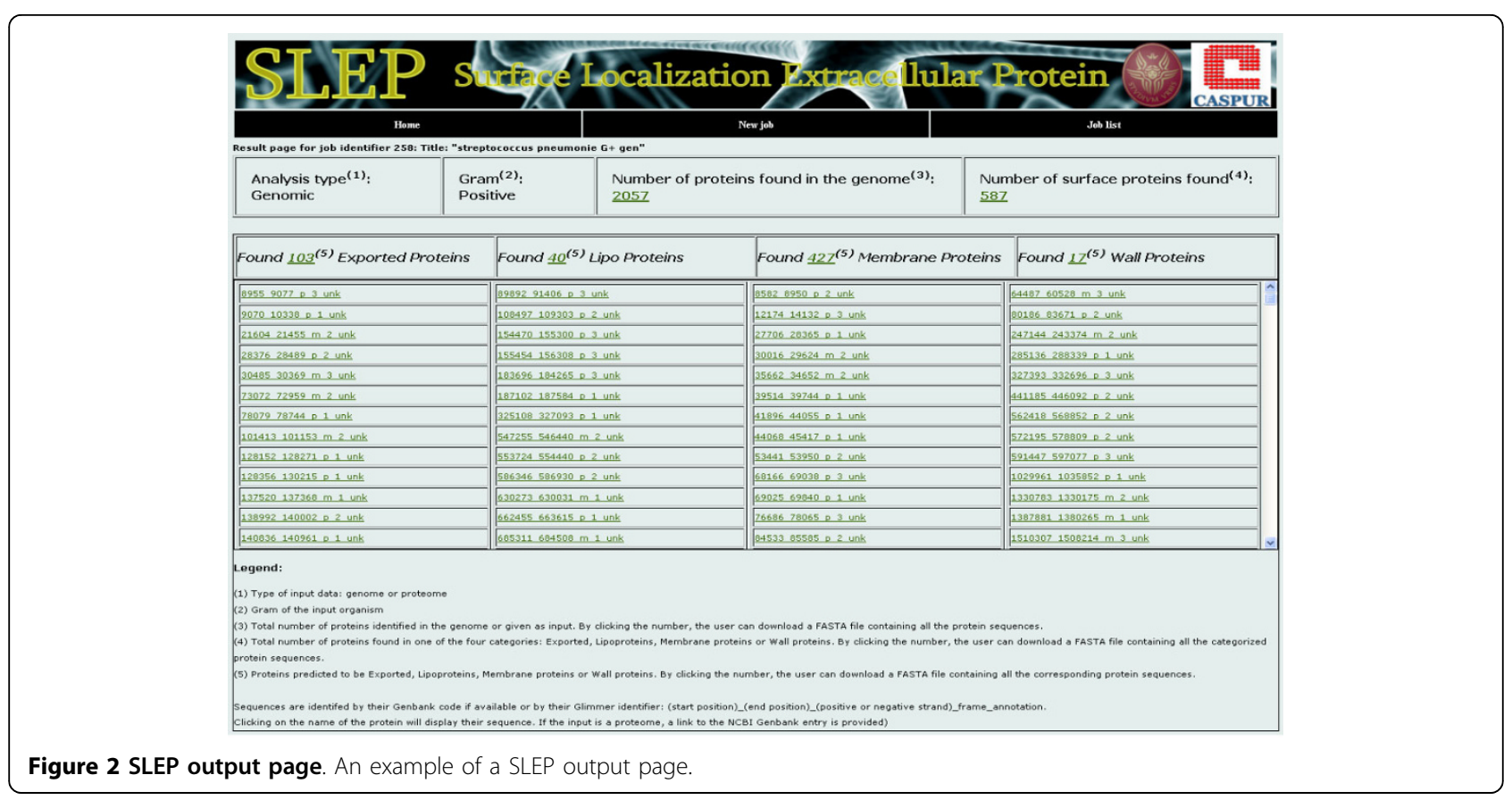

Table 2 SLEP overall accuracy

\begin{tabular}{|c|c|c|c|c|}
\hline & \multicolumn{4}{|c|}{ Gram+ Total number of proteins: 9,564} \\
\hline & MEMBRANE & LIPOPROTEINS & EXPORTED & CELL WALL \\
\hline $\mathrm{TP}$ & 2057 & 227 & 279 & 118 \\
\hline$\overline{\mathrm{FP}}$ & 76 & 19 & 221 & 29 \\
\hline$\overline{\mathrm{TN}}$ & 7335 & 9313 & 8960 & 9396 \\
\hline$\overline{\mathrm{FN}}$ & 96 & 5 & 104 & 21 \\
\hline$\overline{\mathrm{SE}}$ & 95.5 & 97.8 & 72.8 & 84.9 \\
\hline$\overline{\mathrm{SP}}$ & 99.0 & 99.8 & 97.6 & 99.7 \\
\hline PPV & 96.4 & 92.3 & 55.8 & 80.3 \\
\hline$\overline{N P V}$ & 98.7 & 99.9 & 98.9 & 99.8 \\
\hline Accuracy & 98.2 & 99.7 & 96.6 & 99.5 \\
\hline \multirow[t]{3}{*}{$\overline{C C M}$} & 94.8 & 94.9 & 62.0 & 82.3 \\
\hline & \multicolumn{4}{|c|}{ Gram-. Total number of proteins: 9,946} \\
\hline & MEMBRANE & LIPOPROTEINS & EXPORTED & OUTER MEMBRANE \\
\hline$\overline{\mathrm{TP}}$ & 2743 & 266 & 711 & 179 \\
\hline$\overline{\mathrm{FP}}$ & 57 & 40 & 224 & 46 \\
\hline $\mathrm{TN}$ & 6971 & 9635 & 8831 & 9696 \\
\hline$\overline{F N}$ & 175 & 5 & 180 & 25 \\
\hline$\overline{\mathrm{SE}}$ & 94.0 & 98.2 & 80.2 & 87.7 \\
\hline$\overline{S P}$ & 99.2 & 99.6 & 97.5 & 99.5 \\
\hline PPV & 98.0 & 86.9 & 75.9 & 79.6 \\
\hline NPV & 97.6 & 99.9 & 98.1 & 99.7 \\
\hline Accuracy & 97.7 & 99.5 & 96.0 & 99.3 \\
\hline$\overline{C C M}$ & 94.3 & 92.1 & 75.8 & 83.2 \\
\hline
\end{tabular}

Overall accuracy of SLEP for Gram+ and Gram- bacteria. See Material and Methods for the definition of the parameters. 
Table 3 Comparison between SLEP and other available tools

\begin{tabular}{|c|c|c|c|c|c|c|c|c|c|}
\hline & \multicolumn{4}{|c|}{ Gram+ } & \multicolumn{5}{|c|}{ Gram- } \\
\hline & SLEP & PSORTb & PHOBIUS & PRED-LIPO & SLEP & PSORTb & PHOBIUS & PRED-LIPO & ProfTMB \\
\hline Membrane & 98.6 & 91.8 & & & 97.8 & 92.7 & & & \\
\hline Lipoproteins & 99.8 & 98.5 & & 99.4 & 99.6 & 98.4 & & 98.9 & \\
\hline Exported & 96.6 & 97.8 & 93.8 & & 95.7 & 94.9 & 90.5 & & \\
\hline Cell wall/Outer membrane & 99.7 & 99.6 & & & 99.5 & 98.9 & & & 97.8 \\
\hline
\end{tabular}

Comparison of the accuracy of SLEP, PSORTb [3132], PHOBIUS [333435], PRED-LIPO [36] and Prof-TMB [3738] on our dataset. For Prof-TMB, we only considered as positive proteins for which at least 14 transmembrane segments were predicted as this choice substantially increases the accuracy of the method.

for their availability as stand-alone programs since they are all implemented locally to speed up the procedure.

\section{Conclusions}

Bioinformatics tools are extremely useful for the bench scientists and most of them are mature enough to be considered part of a toolbox that should be readily and easily accessible to all.

The appropriate usage of the tools is however essential. This is far from being trivial: one of the most cogent problems in bioinformatics is that way too often obsolete tools remain available and are used by experimentalists who are unaware of more recent developments. Users are confronted with too many available tools, not all properly benchmarked and updated and this can result in a waste of time and effort. The problem is even more relevant when the methods need to be used as start points of a set of experiments where an incorrect selection/usage of the methods can seriously affect the end results.

The initial selection of the set of transcripts/proteins from a pathogen to be used as targets for the development of vaccines and/or inhibitor screening is one such case and yet no comprehensive easy-to-use system was available so far. Perhaps the most complete resource available is Augur [39] which includes a precompiled list of protein localizations and other useful features, but does not allow users to supply their own genome/proteome or set of proteins as is the case in SLEP and is limited to Gram negative bacteria.

We have described here an automatic procedure designed to achieve an accurate prediction of bacterial protein localization via an appropriate sequence of usage of the available methods that is, at the same time, extremely easy to use.

SLEP uses a combination of state of the art methods that have been shown to be the most accurate available $[29,30,32]$. The specific order of usage of these programs has been designed to reduce the chance of misclassification by each of the tools.

The system relieves the bench scientists from the burden of selecting the most accurate programs for the task at hand. SLEP will be continuously updated to reflect novel developments and plans to be the one-stop shop for the analysis of bacterial protein localization that is perhaps the most important aspect of therapeutic target selection.

\section{Availability and requirements}

- Project name: SLEP

- Project home page: http://www.caspur.it/slep

- Operating system(s): Platform independent

- Programming language: Perl and Python

- Any restrictions to use by non-academics: None

\section{Acknowledgements}

This work is supported by the FIRB projects ITALBIONET, Proteomica and by the Italian Ministry of Labour, Health and Social Policies contract no.onc_ord 25/07.

\section{Author details}

'Department of Biochemical Sciences "A. Rossi Fanelli", Sapienza University. 00185 Rome, Italy. ${ }^{2}$ Center for Advanced Studies, Research and Development in Sardinia (CRS4), 09010 Pula, Italy. ${ }^{3}$ CASPUR, Consorzio Interuniversitario per le Applicazioni di Supercalcolo per Universita' e Ricerca, 00185 Rome, Italy. ${ }^{4}$ Istituto Pasteur Fondazione Cenci - Bolognetti, Sapienza University. 00185 Rome, Italy.

\section{Authors' contributions}

EG implemented the system. MO wrote the scripts for running glimmer. DC designed and developed the SLEP website. AT was involved in coordinating the work and in drafting the manuscript. All authors read and approved the final manuscript

Received: 13 September 2009

Accepted: 20 January 2010 Published: 20 January 2010

\section{References}

1. Cohen R: The need for prudent use of antibiotics and routine use of vaccines. Clin Microbiol Infect 2009, 15(Suppl 3):21-23.

2. Salisbury D, Ramsay M, Noakes K, (eds): Immunisation against infectious disease. London: The Stationery Office, 32006.

3. Balestrazzi P, Banchini G, Menozzi MG, Frassi C, De Angelis GL, Giovannelli G: [Yersinia enterocolitica infection in thalassemia. Report of one case (author's transl)]. Pediatr Med Chir 1981, 3(4):269-271.

4. Obaro SK, Monteil MA, Henderson DC: The pneumococcal problem. Bmj 1996, 312(7045):1521-1525

5. Bergmann $\mathrm{S}$, Hammerschmidt S: Versatility of pneumococcal surface proteins. Microbiology 2006, 152(Pt 2):295-303.

6. Kostiukova NN, Bekhalo VA: [Epidemic process of gonorrhea in modern world]. Zh Mikrobiol Epidemiol Immunobiol 2009, , 1: 87-93.

7. The WHO/IUATLD Global Project on Anti-tuberculosis Drug Resistance Surveillance 2002-2007/[written by Abigail Wright, Matteo Zignol]: Antituberculosis drug resistance in the world: fourth global report. Geneva Switzerland: World Health Organization 2008, WHO/HTM/TB/2008.394. 
8. Lindahl G, Stalhammar-Carlemalm M, Areschoug T: Surface proteins of Streptococcus agalactiae and related proteins in other bacterial pathogens. Clin Microbiol Rev 2005, 18(1):102-127.

9. Lin J, Huang S, Zhang Q: Outer membrane proteins: key players for bacterial adaptation in host niches. Microbes Infect 2002, 4(3):325-331.

10. Niemann HH, Schubert WD, Heinz DW: Adhesins and invasins of pathogenic bacteria: a structural view. Microbes Infect 2004, 6(1):101-112.

11. Galperin MY, Koonin EV: Searching for drug targets in microbial genomes. Curr Opin Biotechnol 1999, 10(6):571-578.

12. Pizza $M$, et al: Identification of vaccine candidates against serogroup $B$ meningococcus by whole-genome sequencing. Science 2000, 287(5459):1816-1820.

13. Maione D, Margarit I, Rinaudo CD, Masignani V, Mora M, Scarselli M, Tettelin H, Brettoni C, lacobini ET, Rosini R, D'Agostino N, Miorin L, Buccato S, Mariani M, Galli G, Nogarotto R, Nardi Dei V, Vegni F, Fraser C, Mancuso G, Teti G, Madoff LC, Paoletti LC, Rappuoli R, Kasper DL, Telford JL, Grandi G: Identification of a universal Group B streptococcus vaccine by multiple genome screen. Science 2005, 309(5731):148-150.

14. Rodriguez-Ortega MJ, Norais N, Bensi G, Liberatori S, Capo S, Mora M, Scarselli M, Doro F, Ferrari G, Garaguso I, Maggi T, Neumann A, Covre A, Telford JL, Grandi G: Characterization and identification of vaccine candidate proteins through analysis of the group A Streptococcus surface proteome. Nat Biotechnol 2006, 24(2):191-197.

15. Ton-That $H$, Marraffini LA, Schneewind O: Protein sorting to the cell wall envelope of Gram-positive bacteria. Biochim Biophys Acta 2004, 1694(13):269-278.

16. Janulczyk R, Rasmussen M: Improved pattern for genome-based screening identifies novel cell wall-attached proteins in gram-positive bacteria. Infect Immun 2001, 69(6):4019-4026.

17. Schulz GE: The structure of bacterial outer membrane proteins. Biochim Biophys Acta 2002, 1565(2):308-317.

18. Molloy MP, Herbert BR, Slade MB, Rabilloud T, Nouwens AS, Williams KL, Gooley AA: Proteomic analysis of the Escherichia coli outer membrane. Eur J Biochem 2000, 267(10):2871-2881.

19. Phadke ND, Molloy MP, Steinhoff SA, Ulintz PJ, Andrews PC, Maddock JR: Analysis of the outer membrane proteome of Caulobacter crescentus by two-dimensional electrophoresis and mass spectrometry. Proteomics 2001, 1(5):705-720

20. Molloy MP, Phadke ND, Chen H, Tyldesley R, Garfin DE, Maddock JR, Andrews PC: Profiling the alkaline membrane proteome of Caulobacter crescentus with two-dimensional electrophoresis and mass spectrometry. Proteomics 2002, 2(7):899-910.

21. Nouwens AS, Cordwell SJ, Larsen MR, Molloy MP, Gillings M, Willcox MD, Walsh BJ: Complementing genomics with proteomics: the membrane subproteome of Pseudomonas aeruginosa PAO1. Electrophoresis 2000, 21(17):3797-3809.

22. Rhomberg TA, Karlberg O, Mini T, Zimny-Arndt U, Wickenberg U, Rottgen M, Jungblut PR, Jeno P, Andersson SG, Dehio C: Proteomic analysis of the sarcosine-insoluble outer membrane fraction of the bacterial pathogen Bartonella henselae. Proteomics 2004, 4(10):3021-3033.

23. Sabarth N, Lamer S, Zimny-Arndt U, Jungblut PR, Meyer TF, Bumann D: Identification of surface proteins of Helicobacter pylori by selective biotinylation, affinity purification, and two-dimensional gel electrophoresis. J Biol Chem 2002, 277(31):27896-27902.

24. Delcher AL, Bratke KA, Powers EC, Salzberg SL: Identifying bacterial genes and endosymbiont DNA with Glimmer. Bioinformatics 2007, 23(6):673-679.

25. Delcher AL, Harmon D, Kasif S, White O, Salzberg SL: Improved microbial gene identification with GLIMMER. Nucleic Acids Res 1999, 27(23):4636-4641.

26. Salzberg $S L$, Delcher AL, Kasif $S$, White O: Microbial gene identification using interpolated Markov models. Nucleic Acids Res 1998, 26(2):544-548.

27. Krogh A, Larsson B, von Heijne G, Sonnhammer EL: Predicting transmembrane protein topology with a hidden Markov model: application to complete genomes. J Mol Biol 2001, 305(3):567-580.

28. Sonnhammer EL, von Heijne G, Krogh A: A hidden Markov model for predicting transmembrane helices in protein sequences. Proc Int Conf Intell Syst Mol Biol 1998, 6:175-182.

29. Viklund $\mathrm{H}$, Elofsson $\mathrm{A}$ : Best alpha-helical transmembrane protein topology predictions are achieved using hidden Markov models and evolutionary information. Protein Sci 2004, 13(7):1908-1917.
30. Juncker AS, Willenbrock $H$, Von Heijne $G$, Brunak $S$, Nielsen $H$, Krogh A: Prediction of lipoprotein signal peptides in Gram-negative bacteria. Protein Sci 2003, 12(8):1652-1662.

31. Gardy JL, Laird MR, Chen F, Rey S, Walsh CJ, Ester M, Brinkman FS: PSORTb v.2.0: expanded prediction of bacterial protein subcellular localization and insights gained from comparative proteome analysis. Bioinformatics 2005, 21(5):617-623.

32. Gardy JL, Spencer C, Wang K, Ester M, Tusnady GE, Simon I, Hua S, deFays K, Lambert C, Nakai K, Brinkman FS: PSORT-B: Improving protein subcellular localization prediction for Gram-negative bacteria. Nucleic Acids Res 2003, 31(13):3613-3617.

33. Kall L, Krogh A, Sonnhammer EL: An HMM posterior decoder for sequence feature prediction that includes homology information. Bioinformatics 2005, 21(Suppl 1):i251-257.

34. Kall L, Krogh A, Sonnhammer EL: Advantages of combined transmembrane topology and signal peptide prediction-the Phobius web server. Nucleic Acids Res 2007, , 35 Web Server: W429-432.

35. Kall L, Krogh A, Sonnhammer EL: A combined transmembrane topology and signal peptide prediction method. J Mol Biol 2004, 338(5):1027-1036

36. Bagos PG, Tsirigos KD, Liakopoulos TD, Hamodrakas SJ: Prediction of lipoprotein signal peptides in Gram-positive bacteria with a Hidden Markov Model. J Proteome Res 2008, 7(12):5082-5093.

37. Bigelow HR, Petrey DS, Liu J, Przybylski D, Rost B: Predicting transmembrane beta-barrels in proteomes. Nucleic Acids Res 2004, 32(8):2566-2577.

38. Bigelow H, Rost B: PROFtmb: a web server for predicting bacterial transmembrane beta barrel proteins. Nucleic Acids Res 2006, 34 Web Server: W186-188.

39. Billion A, Ghai R, Chakraborty T, Hain T: Augur-a computational pipeline for whole genome microbial surface protein prediction and classification. Bioinformatics 2006, 22(22):2819-2820.

doi:10.1186/1471-2105-11-39

Cite this article as: Giombini et al:: An automatic method for identifying surface proteins in bacteria: SLEP. BMC Bioinformatics 2010 11:39.

\section{Submit your next manuscript to BioMed Central and take full advantage of:}

- Convenient online submission

- Thorough peer review

- No space constraints or color figure charges

- Immediate publication on acceptance

- Inclusion in PubMed, CAS, Scopus and Google Scholar

- Research which is freely available for redistribution 